\title{
Rapid determination of cutoff rigidities and asymptotic directions using predetermined data from a database
}

\author{
Rolf Bütikofer* and Erwin Flückiger \\ Physikalisches Institut, University of Bern / HFSJG, Bern, Switzerland \\ E-mail: rolf.buetikoferespace.unibe.ch, \\ erwin.flueckigerespace.unibe.ch
}

\author{
Dennis Galsdorf, Bernd Heber, Konstantin Herbst, and Christian Steigies \\ Institut für Experimentelle und Angewandte Physik / Extraterrestrial Physics, \\ Christian-Albrechts-Universität, Kiel, Germany \\ E-mail: galsdorfaphysik.uni-kiel.de, heberaphysik.uni-kiel.de, \\ herbstaphysik.uni-kiel.de steigies@physik.uni-kiel.de
}

\begin{abstract}
The investigation of solar cosmic ray events based on neutron monitor measurements requires detailed knowledge about the trajectories of charged particles in the Earth's magnetic field. This information is needed with a high time resolution and for the respective level of disturbance of the geomagnetic field. The determination of cutoff rigidities and asymptotic directions by the standard technique of trajectory computations is a time-consuming process. Furthermore, the magnetic field controlling the transport of charged particles near Earth is dynamic and exhibits variations on different time scales ranging from minutes to millennia. Today's space weather applications request computations in near real-time. Therefore it is desirable to compute trajectories of cosmic ray particles in the magnetic field of the Earth for various scenarios in advance and to store data to describe cutoff rigidities and asymptotic directions at the locations of the neutron monitors of the worldwide network in a database for quick computation. In this work we first investigate in an exemplary way for selected neutron monitor stations how the cutoff rigidities and the asymptotic directions vary with time through day and year, and for different levels of geomagnetic field disturbances. In a second step we then discuss possibilities how the respective information can be stored in a database for a rapid determination of cutoff rigidities and asymptotic directions of selected neutron monitor stations.
\end{abstract}

The 34th International Cosmic Ray Conference,

30 July- 6 August, 2015

The Hague, The Netherlands

\footnotetext{
* Speaker.
} 


\section{Introduction}

The worldwide network of neutron monitors (NMs) is an excellent tool for many space weather applications. The ensemble of NMs together with the geomagnetic field acts as a giant spectrometer and enables the determination of the spectral variations of the galactic cosmic rays near Earth and the spectral characteristics of sporadic solar cosmic ray events in the energy range of $\sim 500 \mathrm{MeV}$ to $\sim 15 \mathrm{GeV}$. In addition, information about the anisotropy of the cosmic ray flux near Earth can be derived, as the viewing directions of each NM station at the border of the geomagnetosphere depend on the NM location and on the cosmic ray particle's rigidity. The effect of the geomagnetic field on the transport of cosmic ray particles is characterised by cutoff rigidities and by asymptotic directions. The cutoff rigidity describes the shielding by the Earth's magnetic field against the incidence of charged cosmic ray particles (for definitions of cutoff rigidities see [1]) and the asymptotic direction of a cosmic ray particle with rigidity, $R$, arriving at a specific location on the Earth from a defined direction of incidence is a vector pointing in the reverse direction of the particle's velocity vector prior to the particle's entry into the geomagnetic field. The cutoff rigidities and the asymptotic directions depend on the NM location, the particle rigidity, the time, the level of disturbance of the geomagnetic field, and on the direction of incidence over the location of the NM. The computation of cosmic ray particle trajectories inside the geomagnetic field is a time-consuming process. The growing demand of real-time space weather applications requires that the characteristics of the particle transport, i.e. cutoff rigidities and asymptotic directions, are available instantly. Therefore it is desirable to compute trajectories of cosmic ray particles in the magnetic field of the Earth for the locations of all operating NM stations in advance and to archive the information in a database for quick determination of cutoff rigidities and asymptotic directions.

In the following sections we present first the results of an investigation of the variations of cutoff rigidities and of asymptotic directions for selected NM stations located at different latitudes (Athens, Irkutsk, Jungfraujoch, Kiel, and McMurdo). The trajectory computations were made with the software MAGNETOCOSMICS [2] with the magnetic field model IGRF [3] describing the geomagnetic main field and the Tsyganenko model 1989 [4] for the magnetic field caused by different electric current systems in the magnetospheric plasma. The computations were made for particles with vertical incidence into the Earth's atmosphere at an altitude of $20 \mathrm{~km}$ asl. We then discuss possibilities to store the information about cutoff rigidities and asymptotic directions of the NM stations in a suitable form in a database for rapid use.

\section{The daily variation of asymptotic directions}

Due to the daily rotation of the Earth within the geomagnetosphere the asymptotic longitudes and latitudes show a daily variation. Figure 1 shows the asymptotic latitude and longitude values for vertical direction of incidence above the $\mathrm{NM}$ station Kiel $\left(54.30^{\circ} \mathrm{N}, 10.10^{\circ} \mathrm{E}\right)$ for the rigidities 2.5, 3.0, 4.0, and 5.0 GV over a period of three successive days (2010-01-01 00:00 - 2010-0103 24:00 UT) for undisturbed geomagnetic conditions, i.e. $K_{p}=0$. It is obvious that both the asymptotic latitude as well as the asymptotic longitude show a daily variation which is more pronounced at low rigidities. The maximum change in the asymptotic direction in the course of one day is almost $20^{\circ}$ in latitude and almost $40^{\circ}$ in longitude for particles with a rigidity of $2.5 \mathrm{GV}$. 

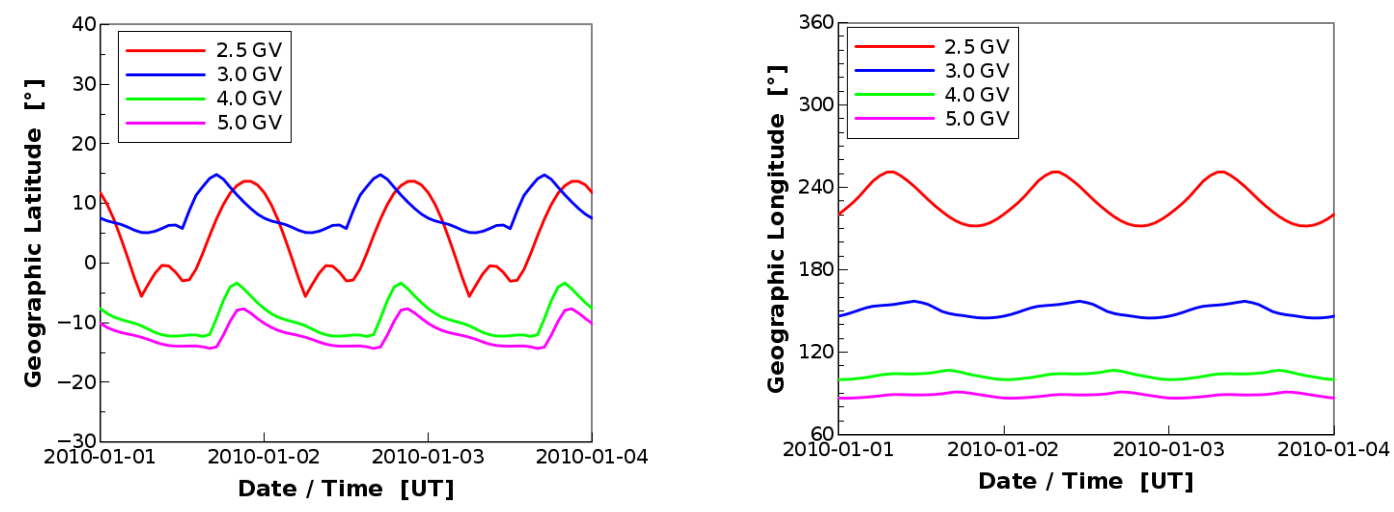

Figure 1: Asymptotic latitude (left) and longitude (right) for the period 2010-01-01 00:00 UT until 201001-03 24:00 UT for cosmic ray particles of 2.5, 3.0, 4.0, and 5.0 GV respectively arriving from the vertical direction at the location of the NM station Kiel for an undisturbed geomagnetic field $\left(K_{p}=0\right)$.

Figure 2 shows the asymptotic geographic latitudes and longitudes as function of rigidity for different times on 2010-01-01 (00:00, 06:00, 12:00, 18:00, and 24:00 UT). The first discontinuity rigidity, $R_{1}$, i.e. the rigidity associated with the first discontinuity in asymptotic longitude occurring as the trajectory calculations are progressing down through the rigidity spectrum, for the Kiel NM location varies in the course of one day between $\sim 2.39-2.45 \mathrm{GV}$ as indicated in the right panel of the figure by vertical arrows. For Irkutsk $\left(52.47^{\circ} \mathrm{N}, 104.03^{\circ} \mathrm{E}\right)$ the daily variation of $R_{1}$ on 2010 01-01 is 3.49-3.54 GV, 4.82-4.85 GV for Jungfraujoch $\left(46.55^{\circ} \mathrm{N}, 7.98^{\circ} \mathrm{E}\right)$, and $8.65-8.69 \mathrm{GV}$ for Athens $\left(37.98^{\circ} \mathrm{N}, 23.78^{\circ} \mathrm{E}\right)$.

For rigidities below $R_{1}$ the asymptotic directions show no longer a continuous trend of change. The asymptotic latitude and longitude show an almost random behaviour. This so-called penumbra region, i.e. the region between the Störmer and main cutoff rigidity [1] consists of alternate rigidity bands of allowed and forbidden trajectories. The relevance of the penumbra for NM data analysis is discussed in section 5 .
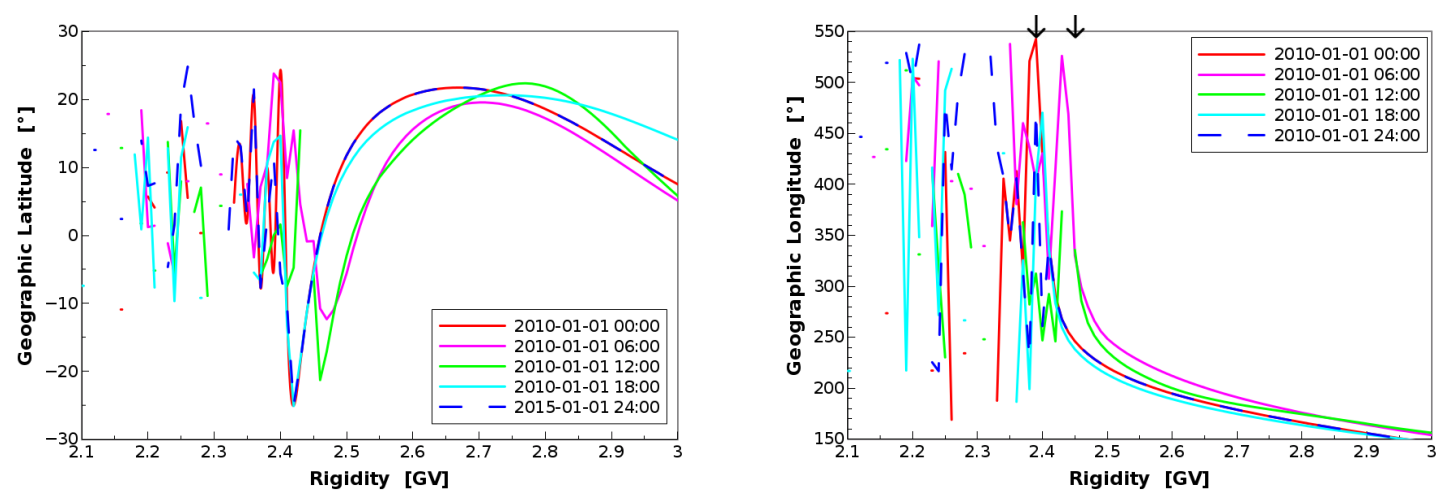

Figure 2: Asymptotic latitude (left) and longitude (right) as function of the particle's rigidity for different times on 2010-01-01 (00:00, 06:00, 12:00, 18:00, and 24:00 UT) for the location of the NM station Kiel for an undisturbed geomagnetic field $\left(K_{p}=0\right)$. The vertical arrows indicate minimum and maximum $R_{1}$. 


\begin{tabular}{|l|ccccccc|}
\hline NM station & \multicolumn{7}{|c|}{$R_{1}[\mathrm{GV}]$} \\
& $K_{p}=0$ & $K_{p}=1$ & $K_{p}=2$ & $K_{p}=3$ & $K_{p}=4$ & $K_{p}=5$ & $K_{p}=6$ \\
\hline McMurdo & 0.00 & 0.00 & 0.00 & 0.00 & 0.00 & 0.00 & 0.00 \\
Kiel & 2.38 & 2.36 & 2.31 & 2.30 & 2.24 & 2.15 & 2.09 \\
Irkutsk & 3.53 & 3.52 & 3.51 & 3.50 & 3.48 & 3.41 & 3.34 \\
Jungfraujoch & 4.83 & 4.81 & 4.79 & 4.76 & 4.72 & 4.64 & 4.59 \\
Athens & 8.68 & 8.66 & 8.63 & 8.61 & 8.57 & 8.49 & 8.43 \\
\hline
\end{tabular}

Table 1: The first discontinuity rigidity, $R_{1}$, as function of $K_{p}$ for selected NM stations for 2010-0101 00:00 UT.

\section{The influence of geomagnetic disturbed conditions on cutoff rigidities and asymptotic directions}

The first discontinuity rigidities, $R_{1}$, for different levels of geomagnetic activity are listed in Table 1 for the NM stations considered in this work for 2010-01-01 00:00 UT.

Figure 3 shows the asymptotic latitude and longitude for different levels of geomagnetic activity for cosmic ray particles with rigidity $1 \mathrm{GV}$ at the $\mathrm{NM}$ station McMurdo $\left(77.51^{\circ} \mathrm{S}, 166.43^{\circ} \mathrm{E}\right)$ during the time period 2010-01-01 00:00 UT - 2010-01-03 24:00 UT. With increasing disturbance of the geomagnetic field also the variations in the asymptotic directions in the course of a day are increasing.
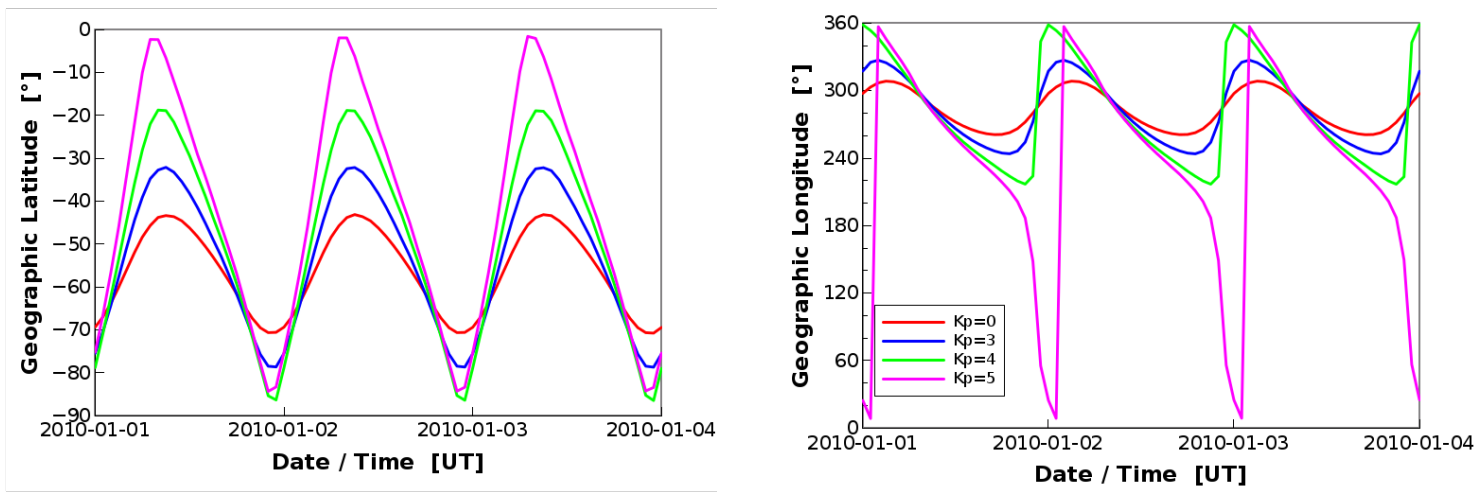

Figure 3: Asymptotic latitude (left) and longitude (right) for the time period 2010-01-01 00:00 UT - 201001-03 24:00 UT for cosmic ray particles with rigidity $1 \mathrm{GV}$ arriving from the vertical direction above the location of the NM station McMurdo for different levels of geomagnetic field disturbance: $K_{p}=0,3,4,5$.

For the determination of the change in asymptotic directions above $R_{1}$ during periods of enhanced geomagnetic activity Flueckiger et al. [5] have developed a simple procedure. According to this procedure the asymptotic latitude $\lambda^{\prime}(R)$ and longitude $\phi^{\prime}(R)$ for a time with enhanced geomagnetic activity can be estimated with the following formulas:

$$
\begin{gathered}
\lambda^{\prime}(R) \approx \lambda\left(R-\Delta R_{1}\right) \\
\phi^{\prime}(R) \approx \phi\left(R-\Delta R_{1}\right)+S \cdot \Delta R_{1}
\end{gathered}
$$


where $\lambda(R)$ and $\phi(R)$ are the asymptotic latitude and longitude in the geographic coordinate system for cosmic ray particles with rigidity $R$ for vertical incidence at the selected NM station for a quiet geomagnetic field, i.e. $K_{p}=0 . R_{1}$ is the first discontinuity rigidity for the selected location for times with no geomagnetic activity, and $R_{1}^{\prime}$ is the first discontinuity rigidity at this same location for enhanced geomagnetic activity, and $\Delta R_{1}$ is the difference $R_{1}^{\prime}-R_{1}$. The quantity $S$ is a measure of the residual change in longitude. For low- and mid-latitude locations, $S$ has an average value of about $20^{\circ} / \mathrm{GV}$.

The asymptotic directions in function of rigidity, $R$, for vertically incident cosmic ray particles above the location of the Kiel NM for $K_{p}=5$ as computed with MAGNETOCOSMICS [2] and as derived for $K_{p}=5$ with the model by Flueckiger et al. [5] based on the computations with MAGNETOCOSMICS for $K_{p}=0$ are plotted in Fig. 4. In the high rigidity range the resulting asymptotic directions in latitude and longitude agree well. With decreasing rigidities the difference between the two curves increases significantly and becomes larger than $10^{\circ}$ below $\sim 3 \mathrm{GV}$ for asymptotic latitude and below $\sim 4 \mathrm{GV}$ for asymptotic longitude (the same threshold values are valid for $K_{p}=6$ ). At the other NM stations investigated in this work the rigidity under which the deviation in the asymptotic directions between the two methods becomes larger than $10^{\circ}$ are at higher rigidities, typically $1-2 \mathrm{GV}$ above $R_{1}$ of the corresponding NM station.
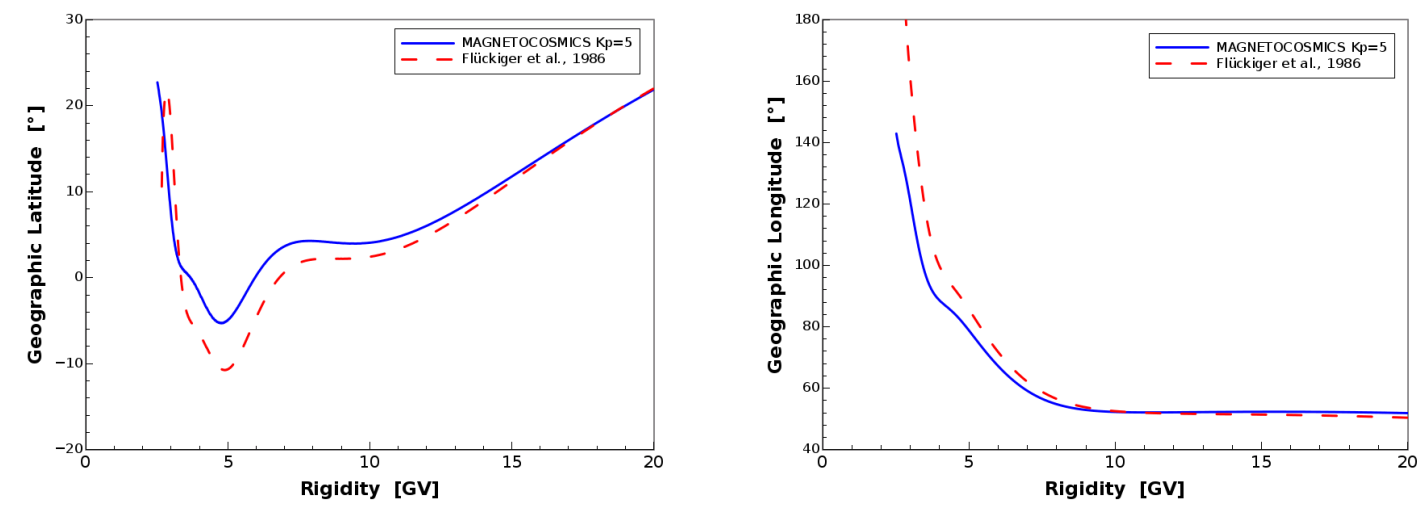

Figure 4: Asymptotic latitude (left) and longitude (right) as function of rigidity at the location of the NM station Kiel for $K_{p}=5$ as computed with MAGNETOCOSMICS (blue, solid lines) and as derived for $K_{p}=5$ with the model by Flueckiger et al. [5] based on the MAGNETOCOSMICS computations with $K_{p}=0$ (red, dashed lines).

\section{Seasonal effects on the asymptotic directions}

The investigation of the variations of the asymptotic directions over different seasons during one year does not show effects larger than $10^{\circ}$ in the asymptotic latitudes and longitudes down to $R_{1}$ for undisturbed geomagnetic conditions. However with an increasing level of geomagnetic activity also the seasonal variations in the asymptotic directions are increasing and must be considered in NM data analysis. As an example the seasonal variations of the asymptotic directions at the NM station McMurdo for rigidity $R=1.0 \mathrm{GV}$ and with high geomagnetic activity $\left(K_{p}=5\right)$ are presented in Fig. 5. The figure shows the results of trajectory computations executed for the time 00:00 UT on the days 1, 10, and 20 of each month during the year 2010. 

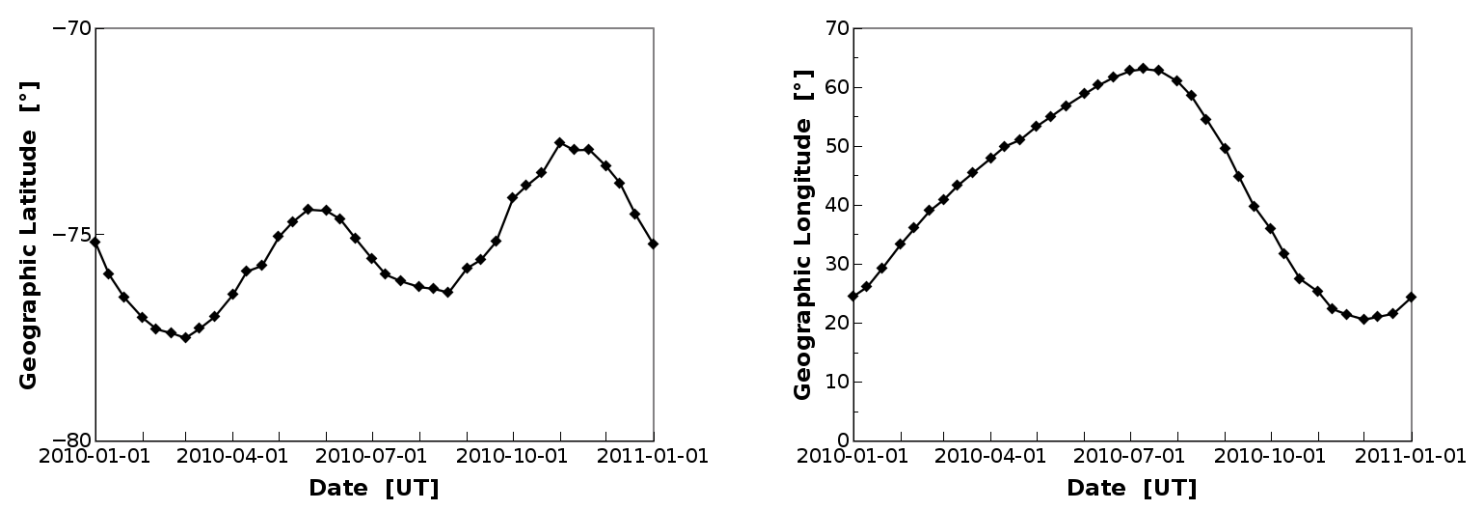

Figure 5: Asymptotic latitude (left) and longitude (right) for vertically incident cosmic ray particles with $R=1.0 \mathrm{GV}$ above the NM station McMurdo with a geomagnetic disturbance $K_{p}=5$.

\section{Penumbra}

The contribution of the penumbra to the count rate of a NM depends on the width of the penumbra, on the shape of the cosmic ray spectrum, and on the particle anisotropy. For the analysis of galactic cosmic rays and of the isotropic phase of solar cosmic ray events the penumbra is usually accounted for by the use of the effective cutoff rigidity, $R_{c}^{e f f}$ [1]. For marked particle anisotropies the penumbra region must be considered in the analysis of NM data, as the contribution from this rigidity range to the counting rate may be considerable at some NM stations. The width of the penumbra is increasing with increasing $R_{1}$ until $\sim 10 \mathrm{GV}$ where it becomes $\sim 2 \mathrm{GV}$ and shows a fast decrease for $R_{1}>10 G V$ with increasing $R_{1}$ [6]. For stations at high latitudes there is practically no penumbra or the penumbra is only small and the contribution of the penumbra region to the NM count rate is usually marginal. The penumbra may be significant, however, for NM stations at mid-latitudes.

Figure 6 shows the asymptotic latitude and longitude vs. rigidity in the penumbra range at the location of the Irkutsk NM for 00:00 and 12:00 UT on 2010-01-01 and for $K_{p}=0$. The spread in asymptotic latitude is confined between $\sim 20^{\circ} \mathrm{S}$ and $\sim 20^{\circ} \mathrm{N}$. For all considered NM stations in this work and for all geomagnetic activity levels, the distribution of the asymptotic latitudes in the penumbra is in the range $\sim 30^{\circ} \mathrm{S}-\sim 30^{\circ} \mathrm{N}$. In contrast the distribution in longitude shows a spread over all geographic longitudes, i.e. from $0^{\circ}$ to $360^{\circ}$. From Fig. 6 it can be seen that there are bands of allowed and forbidden rigidities. These penumbral structures are plotted in Fig. 7 for the NM stations Irkutsk and Jungfraujoch.

\section{Storing of the data into a database}

The following procedure for the predetermination of the cutoff rigidities and the asymptotic directions for the storing in a database is suggested. The cosmic ray particle trajectories are computed e.g. with MAGNETOCOSMICS [2] for all NM stations in operation, for vertical incidence into the Earth's atmosphere, and for the different levels of geomagnetic activity, i.e. $K_{p}=0,1,2,3,4,5,6$. The trajectory calculations are made for the rigidity range from $20 \mathrm{GV}$ down to $0 \mathrm{GV}$ with different step sizes: $1.0 \mathrm{GV}$ between $20 \mathrm{GV}$ and $10 \mathrm{GV}, 0.1 \mathrm{GV}$ between $9.9 \mathrm{GV}$ and $R_{1}+0.2 \mathrm{GV}$, and 

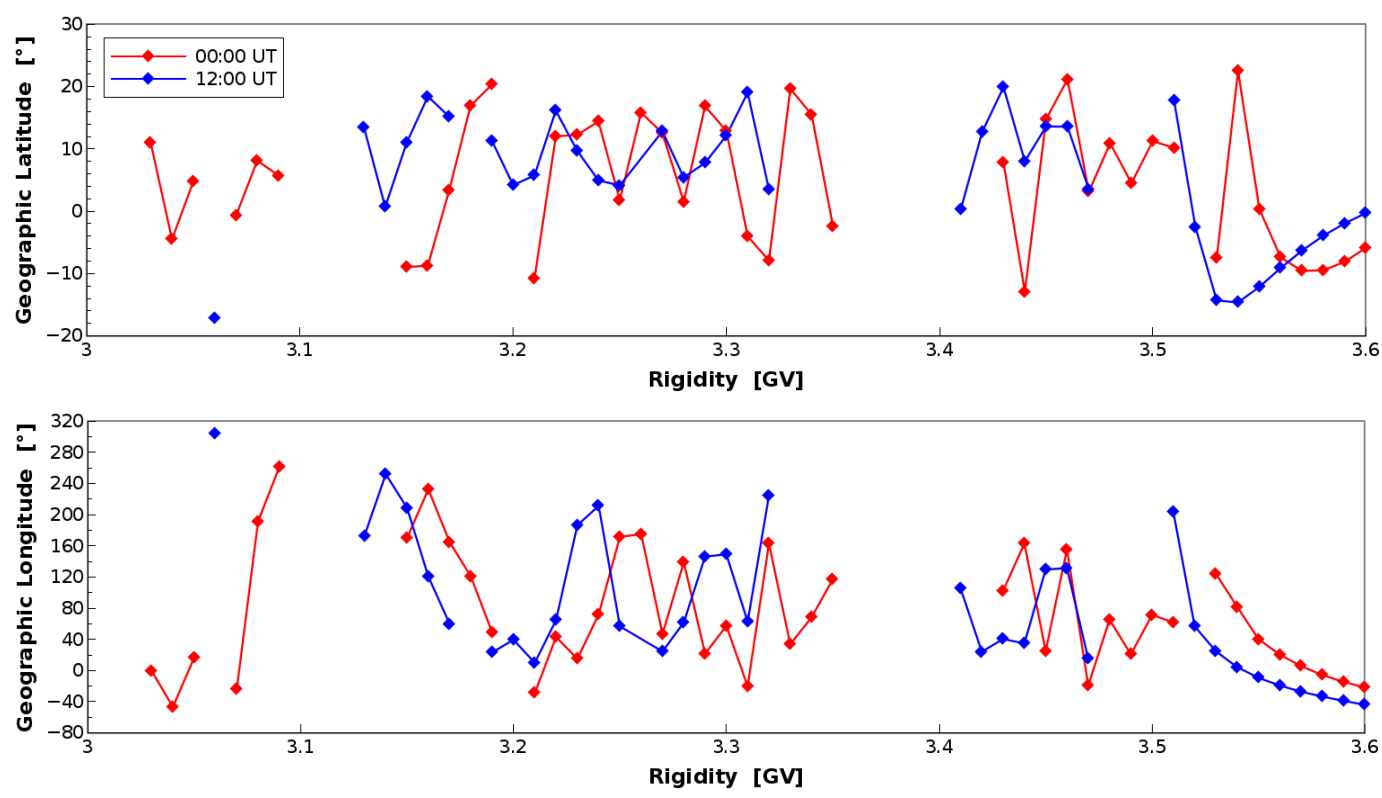

Figure 6: Asymptotic latitude (top) and longitude (bottom) as a function of the rigidity at the location of the NM station Irkutsk over the rigidity range of the penumbra for 00:00 and 12:00 UT on 2010-01-01 and for $K_{p}=0$.
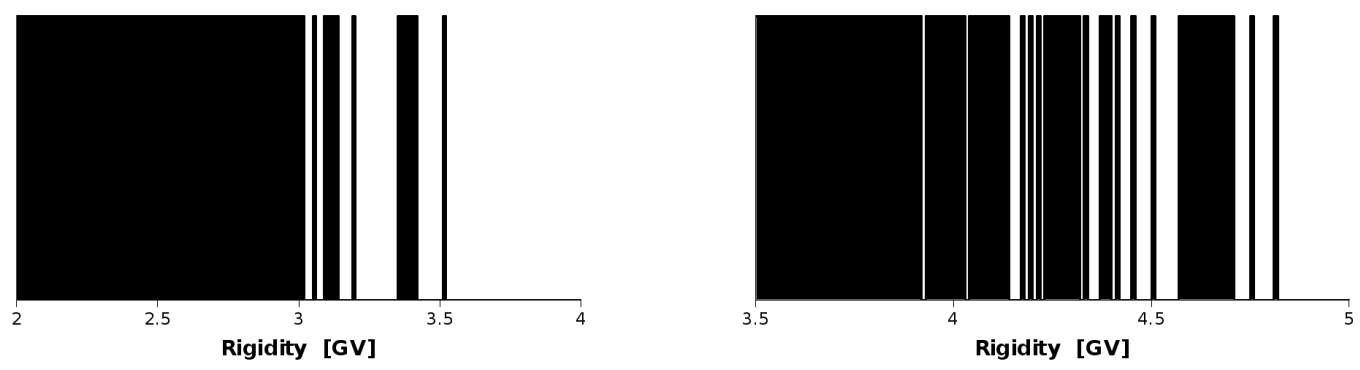

Figure 7: Penumbral structure for cosmic ray particles with vertical incidence into the Earth's atmosphere above Irkutsk (left) and above Jungfraujoch (right) on 2010-01-01 00:00 UT and for $K_{p}=0$. White areas indicate allowed rigidities and black areas indicate forbidden rigidities.

$0.01 \mathrm{GV}$ below $R_{1}+0.2 \mathrm{GV}$. The computations are made for each full hour, but they need not be repeated for each day as the asymptotic latitudes and longitudes show a quasi periodic 24 hour behaviour where the amplitudes change only little over the range of several days. These daily as well as the seasonal effects have to be investigated for each NM station separately and for each level of geomagnetic activity to determine the frequency with which the trajectory computations have to be repeated. Seasonal effects in the asymptotic directions are only dominant at lower rigidities towards the cutoff rigidities.

It seems meaningful to directly archive the computed asymptotic latitudes and longitudes for each NM in the database. From the present status of the investigations it is not yet clear how the information of the allowed trajectories in the rigidity range of the penumbra should be stored in 
the database. Further enquiries for all NMs of the worldwide network are needed to obtain a good solution in this issue.

\section{Conclusions}

The procedure of archiving the information about cutoff rigidities and asymptotic directions in a database in advance to allow a rapid determination in case of a space weather event observed by the worldwide network of NMs is not trivial. The lack of a systematic behaviour of the characteristic parameters for the NMs at different locations and for different geomagnetic activities demands an individual handling.

It is planned that the cutoff rigidities and asymptotic directions will be available to users for freely selected times via a web interface from the NMDB [7] webpage for all active NMs and for geomagnetic activities represented by the $K_{p}$-index.

\section{Acknowledgments}

This project has received funding from the European Union's Horizon 2020 research and innovation program under grant agreement No 637324. RB and EF thank the University of Bern and the International Foundation High Altitude Research Stations Jungfraujoch and Gornergrat for the support.

\section{References}

[1] D. J. Cooke, J. E. Humble, M. A. Shea, D. F. Smart, and N. Lund, On cosmic-ray cut-off terminology, Nuovo Cimento C Geophysics Space Physics C, 14 (213-234) 1991.

[2] L. Desorgher, http://cosray.unibe.ch/laurent/magnetocosmics last access: 25 June 2015, 2004

[3] IAGA Division V, Working Group V-MOD, IGRF Model, http://www.ngdc.noaa.gov/IAGA/vmod/igrf.html last access: 25 June 2015

[4] N. A. Tsyganenko, A magnetospheric magnetic field model with a warped tail current sheet, Planetary and Space Science 37 (5-20) 1989.

[5] E. O. Flueckiger, D. F. Smart, and M. A. Shea, A procedure for estimating the changes in cosmic ray cutoff rigidities and asymptotic directions at low and middle latitudes during periods of enhanced geomagnetic activity, JGR 91 (7925-7930) 1986.

[6] D. F. Smart and M. A. Shea, An Analysis of Trajectory-Derived Penumbral Widths, International Cosmic Ray Conference 4 (1309-1314) 1975.

[7] NMDB: real-time database for high resolution neutron monitor measurements, http://www.nmdb.eu last access: 29 June 2015 